\title{
BMJ Open SCING - Spinal Cord Injury Neuroprotection with Glyburide: a pilot, open-label, multicentre, prospective evaluation of oral glyburide in patients with acute traumatic spinal cord injury in the USA
}

\author{
Amy Janelle Minnema, ${ }^{1}$ A Mehta, ${ }^{2}$ Warren W Boling, ${ }^{3}$ Jan Schwab, ${ }^{4}$
} J Marc Simard, ${ }^{5}$ H Francis Farhadi (i) ${ }^{1}$

To cite: Minnema AJ, Mehta A, Boling WW, et al. SCING—Spinal Cord Injury Neuroprotection with Glyburide: a pilot, open-label, multicentre, prospective evaluation of oral glyburide in patients with acute traumatic spinal cord injury in the USA. BMJ Open 2019;9:e031329. doi:10.1136/ bmjopen-2019-031329

- Prepublication history and additional material for this paper are available online. To view these files, please visit the journal online (http://dx.doi. org/10.1136/bmjopen-2019031329).

Received 29 April 2019 Revised 19 August 2019 Accepted 30 August 2019
Check for updates

(C) Author(s) (or their employer(s)) 2019. Re-use permitted under CC BY-NC. No commercial re-use. See rights and permissions. Published by BMJ.

For numbered affiliations see end of article.

Correspondence to Dr H Francis Farhadi; francis.farhadi@osumc.edu

\section{ABSTRACT}

Introduction Acute traumatic spinal cord injury (tSCl) is a devastating neurological disorder with no pharmacological neuroprotective strategy proven effective to date. Progressive haemorrhagic necrosis (PHN) represents an increasingly well-characterised mechanism of secondary injury after $\mathrm{tSCl}$ that negatively impacts neurological outcomes following acute tSCl. Preclinical studies evaluating the use of the Food and Drug Administration-approved sulfonylurea receptor 1-transient receptor potential melastatin 4 channel blocker glyburide in rodent models have shown reduced secondary microhaemorrhage formation and the absence of capillary fragmentation, the pathological hallmark of PHN.

Methods and analysis In this initial phase multicentre open-label pilot study, we propose to enrol 10 patients with acute cervical $\mathrm{tSCl}$ to primarily assess the feasibility, and safety of receiving oral glyburide within 8 hours of injury. Secondary objectives include pharmacokinetics and preliminary evaluations on neurological recovery as well as blood and MRI-based injury biomarkers. Analysis will be performed using the descriptive and non-parametric statistics.

Ethics and dissemination Glyburide has been shown as an effective neuroprotective agent in preclinical $\mathrm{tSCl}$ models and in the treatment of ischaemic stroke with the additional risk of a hypoglycaemic response. Given the ongoing secondary injury and the traumatic hyperglycaemic stress response seen in patients with tSCl, glyburide; thus, offers an appealing neuroprotective strategy to supplement standard of care treatment. The study protocol was approved by the Ohio State University Biomedical Institutional Review Board. The protocol was amended in February 2017 with changes related to study feasibility and patient recruitment. Specifically, the route of administration was changed to the oral form to allow for streamlined and rapid drug administration, and the injury-to-drug time window was extended to 8 hours in an effort to further enhance enrolment. Participants or legally authorised representatives are informed about the trial and its anticipated risks orally and in written form using
Strengths and limitations of this study

- The Spinal Cord Injury Neuroprotection with Glyburide (SCING) study is the first clinical trial evaluating the sulfonylurea receptor 1 -transient receptor potential melastatin 4 channel blocker glyburide administered acutely after traumatic spinal cord injury (tSCl).

- Rodent tSCI models to date have independently confirmed reduced secondary micro haemorrhage when glyburide is administered up to 3-4 hours after injury, a timeline that approximates the clinically feasible SCING injury-to-drug time window of 8 hours.

- As an initial opel-label pilot phase study, SCING has inherent limitations that include a small sample size and lack of a control group.

- The feasibility and safety of oral glyburide administration within the first 8 hours following cervical $\mathrm{tSCl}$ will be assessed as primary outcome measures.

- The feasibility of undertaking this pilot study will inform a more rational subsequent phase II trial design, including whether exemption from informed consent may be justified in future iterations.

an approved informed consent form prior to inclusion. The findings of this study will be disseminated to the participants and to academic peers through scientific conferences and peer-reviewed journal publications.

Trial registration numbers NCT02524379 and $2014 \mathrm{H} 0335$.

\section{INTRODUCTION}

Acute traumatic spinal cord injury (tSCI) is a devastating neurological disorder with no standard of care neuroprotective strategies proven effective to date. In this initial phase multicentre open-label pilot study, we propose to enrol patients with acute cervical 
tSCI with defined inclusion and exclusion criteria to assess the feasibility and safety of early treatment with oral glyburide.

Oral glyburide (also known as glibenclamide; 5-chloro-N-(4-(N-(cyclohexylcarbamoyl) sulfamoyl) phenethyl)-2-methoxybenzamide) is an antidiabetic sulfonylurea that has been used successfully in the treatment of non-insulin-dependent diabetes mellitus (NIDDM) for more than 30 years. In treating NIDDM, the drug works by inhibiting adenosine triphosphate (ATP)-sensitive potassium channels in pancreatic beta cells by antagonism of sulfonylurea receptor 1 (Sur1). This inhibition causes cell membrane depolarisation, voltage-dependent calcium channel opening and an increase in beta cell intracellular calcium, thus stimulating insulin release.

In the central nervous system (CNS), the Sur1-transient receptor potential melastatin 4 (Trpm4) channel is selectively expressed under conditions of ischaemia, hypoxia and trauma,${ }^{1-4}$ leading to vasogenic and cytotoxic oedema followed by oncotic cell death. ${ }^{5}$ In a rodent tSCI model, Sur1-Trpm4 was first noted to be upregulated at 6 hours with expanding expression noted up to 24 hours following injury. With respect to microvascular endothelial cells, this process leads to the formation of space-occupying oedema and secondary haemorrhage, which are critical secondary injury mechanisms that progressively exacerbate mechanical compression, ischaemia and neuronal/glial cell death following CNS trauma. ${ }^{6}$ Glyburide-mediated blockade of the Sur1-Trpm 4 channel in rodent models of tSCI leads to reduced secondary micro haemorrhage formation, reduced lesion volumes and improved hindlimb locomotor functional outcomes. ${ }^{4-10}$

Given the above, we have set out to assess the feasibility of screening, consenting, enrolling and administering oral glyburide in the acute setting following tSCI $(\leq 8$ hours from injury) at our participating centres. Further, a comprehensive assessment of all adverse events (AEs) will be undertaken. The data obtained from this pilot study will inform the design of subsequent multicentre phase II clinical studies evaluating the safety and efficacy of oral glyburide in potentially improving functional outcomes and surrogate injury biomarkers following acute tSCI.

\section{METHODS AND ANALYSIS}

\section{Study design}

Spinal Cord Injury Neuroprotection with Glyburide (SCING) is a prospective single-armed, open-label, multicentre pilot study designed to assess the safety and feasibility of administering oral glyburide in the acute phase following tSCI. Beginning in June 2017, patients have been consecutively screened with defined inclusion/ exclusion criteria (see box 1) at three level I trauma centres including Ohio State University Wexner Medical Center (Principal Site), University of Illinois-Chicago and Loma Linda University. Screening is planned to extend to June of 2022. Data and regulatory management

\section{Box 1 SCING clinical trial eligibility criteria}

\section{Inclusion}

- Age: $\geq 18$ years and $\leq 80$ years.

- Written informed consent by patient or legal authorised representative.

- No other life-threatening injury.

- No evidence of sepsis.

- Acute cervical tSCI with ASIA Impairment Scale grade A, B or C on admission.

- Non-penetrating tSCl at neurological level from C2 to C8.

- Initiation of study drug within 8 hours of injury.

\section{Exclusion}

- Unconsciousness or other mental impairment that prevents neurological assessment within the first 8 hours.

- Acute tSCI with ASIA Impairment Scale grade D or E.

- Currently involved in another non-observational tSCI research study or receiving another investigational drug.

- History of hypersensitivity to sulfonylureas, in particular glyburide or any of its components.

- Other illness (including mental disorder) that could preclude accurate medical and neurological evaluation (at discretion of the site investigator).

- Unable to commit to the follow-up schedule.

- A recent history of regular substance abuse (illicit drugs and alcohol), which in the opinion of the investigator would interfere with the subject's participation in the study.

- Any condition likely to result in the patient's death within the next 12 months.

- Prisoner.

- Severe renal disorder from the patient's history (eg, dialysis) or baseline eGFR of $<30 \mathrm{~mL} / \mathrm{min} / 1.73 \mathrm{~m}^{2}$.

- Known severe liver disease or ALT >3 times upper limit of normal or bilirubin $>2$ times upper limit normal. Subjects may be randomised if liver function tests have been drawn but are not yet available and the subject has no known history of liver disease; however, treatment with glyburide will be discontinued prior to the second dose if liver function tests indicate ALT>3 times upper limit of normal or bilirubin $>2$ times upper limit of normal.

- Blood glucose $<55 \mathrm{mg} / \mathrm{dL}$ at enrolment or immediately prior to administration of glyburide. or a clinically significant history of hypoglycaemia.

- Acute ST elevation myocardial infarction, and/or acute decompensated heart failure, and/or Q-T interval corrected for heart rate (QTC) $>520 \mathrm{~ms}$, and/or known history of cardiac arrest (pulseless electrical activity (PEA), ventricular tachycardia (VT), ventricular fibrillation (VF), asystole), and/or admission for an acute coronary syndrome, myocardial infarction or coronary intervention (percutaneous coronary intervention or coronary artery surgery) within the past 3 months.

- Known treatment with Bosentan within past 7 days.

- Known G6PD enzyme deficiency.

- Pregnancy: Women must be either postmenopausal, permanently sterilised or, if $\leq 50$ years old, must have a negative test for pregnancy obtained before enrolment.

- Breastfeeding women who do not agree to stop breast feeding during and for 7 days following the end of oral glyburide administration.

- Subjects who in the opinion of the investigator are not suitable for inclusion in the study (reason to be documented).

Continued 


\section{Box 1 Continued}

ALT, alanine transaminase; ASIA, American Spinal Injury Association; eGFR, estimated glomerular filtration rate; SCING, Spinal Cord Injury Neuroprotection with Glyburide; $\mathrm{tSCl}$, traumatic spinal cord injury.

as well as statistical analyses are performed at the principal site. All study investigators are trained and experienced in the assessment and management of patients with tSCI including certifications through the American Spinal Injury Association (ASIA) e-learning Centre. Communication between the three enrolling centres will be facilitated through regular conference calls. All protocol modifications will first be approved through the coordinating centre institutional review board (IRB) and then will be communicated via email to the subsite teams.

\section{Primary objectives}

The primary objectives are to assess the feasibility and safety of undertaking a larger phase II study among this population of patients where treatment must begin within a short injury-to-drug time window. The number of patients who would otherwise be eligible for the study but did not receive study treatment within the 8-hour time frame will be recorded (ratio of recruited vs screened). The rate of recruitment will also be recorded. Additional primary objectives include the safety of enrolling, evaluating and treating acute cervical tSCI patients with oral glyburide. Vital signs, clinical examination findings, ECG and clinical laboratory results will be carefully and continuously monitored during the course of hospitalisation.

AEs will be assessed throughout the course of hospitalisation until postinjury day 28. Serious AEs (SAEs) will be monitored for the entire duration of the study. In addition to presenting a summary of all AEs, where possible, a comparison of initial hospitalisation AEs occurring in study subjects versus those occurring in matched historical controls will be made, as captured in the North American Clinical Trials Network (NACTN) registry, ${ }^{11} 12$ a multicentre database of patients with tSCI designed to establish the natural course of recovery following trauma using standardised and validated acute care and follow-up data. To facilitate this comparison, AEs will be grouped into one of 10 systems according to a modified NACTN chart $^{1112}$ : cardiac, pulmonary, haematology, gastrointestinal/genitourinary, infections, skin, failure of stabilisation, neuropsychiatric, hypoglycaemia/other metabolic and other. These AEs will be described further with respect to the specific name of the event (ie, the 'type' of event; eg, for cardiac events, the type could be bradycardia or other dysrhythmia, cardiac arrest, etc). Severity ('intensity', as per the NACTN chart) as well as relatedness to glyburide will also be assessed. The frequency of AEs, as well as mortality (all cause and cardiac related), will be compared with that of a matched cohort from the NACTN registry. ${ }^{112}$
Specific outcome variables related to assessing blood glucose (BG)-related safety of glyburide are hypoglycaemia (defined as BG $<70 \mathrm{mg} / \mathrm{dL}$ ) and symptomatic hypoglycaemia (defined as hypoglycaemia with investigator-identified hypoglycaemic symptoms). Additional specific outcome variables related to assessing cardiac-related safety of glyburide include incidence/severity of cardiac AEs and cardiac mortality, incidence of QTC of $<500 \mathrm{~ms}$, mean QTc, and mean change in QTc from baseline.

\section{Secondary objectives}

Secondary objectives focus on neurological recovery following tSCI, pharmacokinetics, serum biomarkers and spinal cord lesion imaging analysis. The neurological status of patients will be assessed using the ASIA Impairment Scale (AIS) as assessed by International Standards for Neurological Classification of SCI (ISNCSCI) criteria and the Spinal Cord Independence Measure (SCIM version III) ${ }^{13}$ The motor and sensory subscores of the AIS assessment will be determined on first contact, every day until day 4, at 1, 2, 4, 6 weeks and at 3,6,12 months of follow-up. The SCIM version III will be assessed at 3, 6 and 12 months of follow-up.

Given recent observations on the predictive value of admission imaging biomarkers in predicting neurological recovery at discharge and 1-year follow-up, ${ }^{14}{ }^{15}$ the likelihood of AIS conversion will be calculated for each enrolled subject based on admission imaging measurements of axial-based and sagittal-based T2-weighted signal intensity changes (see table 1 for characteristics of retrospective cohort evaluated at the principal site with corresponding 1-year AIS conversion probabilities). AIS recovery comparisons with tSCI patients enrolled in the NACTN registry will not be attempted given the absence of imaging-based data in this database.

Plasma concentrations will be serially quantified through day 3 following tSCI to evaluate the pharmacokinetics of glyburide in the acute tSCI population. Comparisons will be made to reported levels achieved in healthy patient cohorts. Standard ELISA techniques will be used to measure blood levels of neurofilament light chain, neuron-specific enolase, tau, S100b and glial fibrillar acidic protein levels on admission, at 24 hours and on days 3 and 7 following tSCI to evaluate serum biomarker levels. Comparisons will be made to previously published values observed in non-treated control patients. ${ }^{16-18}$ Finally, spinal cord lesion volume will be analysed using standard sequences (including T1 and T2-weighted images) to assess the extent of the haemorrhagic lesion and surrounding oedema. Patients will be imaged on the day of admission and on day 2 following injury demarcating a defined time window for assessment of post-tSCI lesion expansion. ${ }^{19}$ Volumetric assessments of lesion size (based on manual outlines) will be performed and compared at the two time points by the study neuroradiologist to assess for the progression of intrinsic cord signal changes. 
Table 1 Characteristics of consecutive patients admitted between 2008 and 2015 with cervical tSCls at principal site ${ }^{14}$

n $\%$

Probability of AIS conversion at 1-year follow-up

\begin{tabular}{|c|c|c|c|}
\hline Demographics & & & \\
\hline Sex (male/female) & $78 / 21$ & $78.8 / 2$ & \\
\hline Age (mean, years) & $51.2 \pm$ & & \\
\hline AIS grade on admission & & & \\
\hline A & 33 & 33.3 & \\
\hline B & 8 & 8.1 & \\
\hline C & 28 & 28.3 & \\
\hline D & 30 & 30.3 & \\
\hline Glucose on admission (mg/dL) & 130.2 & & \\
\hline Radiology & & & \\
\hline BASIC & & & \\
\hline 0 & 1 & 1.1 & 0.99997 \\
\hline 1 & 13 & 14.4 & 0.99997 \\
\hline 2 & 37 & 41.1 & 0.91667 \\
\hline 3 & 20 & 22.2 & 0.27273 \\
\hline 4 & 19 & 21.2 & 0.00003 \\
\hline IML length (mm) & $32.1 \pm$ & & \\
\hline$\leq 10$ & 6 & 6.67 & 1.00000 \\
\hline $10.1-20$ & 21 & 23.3 & 0.83333 \\
\hline $20.1-30$ & 23 & 25.6 & 0.81250 \\
\hline $30.1-40$ & 15 & 16.7 & 0.60000 \\
\hline$>40$ & 25 & 27.8 & 0.08333 \\
\hline
\end{tabular}

AIS, ASIA Impairment Scale; ASIA, American Spinal Injury Association; BASIC, Brain and Spinal Injury Center Score; IML, intramedullary lesion; tSCl, traumatic spinal cord injury.

\section{Study population}

All subjects with AIS A, B or C acute tSCIs will be assessed for suitability and prospectively consented and enrolled in the study (example informed consent form; online online supplementary appendix 1). Only cervical level (C2-C8) level injuries in patients aged 18-80 years old will be included. Detailed eligibility criteria are listed in box 1 . The use of immunosuppressive therapy (including methylprednisolone or other corticosteroids) will be discouraged; however, use of such therapy will not require exclusion or discontinuation from the study. To assess the feasibility of recruitment and of the protocol procedures (specifically the $\leq 8$ hours injury-to-drug time frame), the study will plan to enrol and treat 10 patients across all study sites. The use of a small sample size, as planned in SCING, is a well-established practice to assess safety, feasibility and pharmacokinetics, particularly for a repurposed drug with a long-established record..$^{20-24}$

\section{Standard of care management}

Cervical CT studies will reveal the nature of the associated spinal fracture and/or misalignment. Subjects with bilateral cervical facet dislocations or with fracture patterns associated with $>75 \%$ canal compromise will be excluded from the study given that a significant component of their underlying tSCI likely involves direct parenchymal and/or ischaemic mechanisms. ${ }^{25}$ Adherence to acute tSCI management guidelines most recently revised by a consensus panel in $2013^{26}$ will be monitored. These guidelines include avoidance of hypoxia and hypotension with induced hypertension as needed to keep mean arterial pressure $\geq 85 \mathrm{~mm} \mathrm{Hg}$ for up to 7 days. Early intervention with surgical decompression and stabilisation within 24-48 hours (when medically feasible) will be encouraged for all patients ${ }^{27}$ but will ultimately be left to the discretion of the treating surgeon.

The following items will be included as standard of care: vital signs (temperature, blood pressure, pulse, respiration rate and $\mathrm{O}_{2}$ saturation), blood work, CT scans of the spine and head (as indicated) and MRI.

\section{Study-related management}

Non-standard of care blood work will include pharmacokinetic and biomarker analysis (online online supplementary appendix 2). Pharmacokinetic-specific blood samples will be collected at each day from baseline to day 3 (four total blood draws). Biomarker-level blood samples will be collected at baseline, 24 hours, and days 3 and 7. Patients will be closely monitored clinically for all AEs during the first 28 days of the study. SAEs will be 


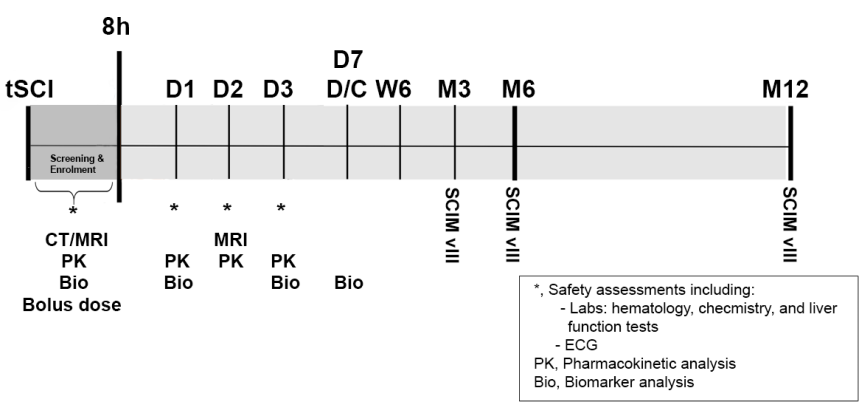

Figure 1 SCING study design. Diagram highlighting research-specific clinical and laboratory testing timeline. ASIA impairment testing is performed at each time point from injury to 1-year follow-up. ASIA, American Spinal Injury Association; SCIM, Spinal Cord Independence Measure; SCING, Spinal Cord Injury Neuroprotection with Glyburide; $\mathrm{tSCl}$, traumatic spinal cord injury.

monitored for the entire duration of the study. Patient-reported pain severity levels will be assessed daily during admission using the Neuropathic Pain Scale $^{28}$ as well as the International Spinal Cord Injury Pain Basic Data Set version 2.0. ${ }^{29}$ Finally, neurological evaluation will be carried out at specific time points up to 1 year following tSCI to assess for changes in neurological status. The study timeline is summarised in figure 1.

\section{Screening and baseline}

Patients with suspected acute cervical tSCI are being screened on arrival to hospital. Evaluations prior to enrolment include cervical tSCI time, mechanism, level of injury and neurological examination including ISNCSCI motor and sensory assessments. Eligible patients are being enrolled as soon as possible after documented tSCI, so that oral glyburide administration can begin no later than 8 hours after injury. This injury-to-drug time frame appears feasible given the injury-to-admission times reported in recent tSCI studies. For instance, in a phase 1/2a Riluzole study, ${ }^{30}$ the mean injury-to-admission and injury-to-drug times were 3.0 and 8.5 hours, respectively, with the latter likely reflecting the time taken for additional MRI in addition to CT imaging.

CT imaging is performed prior to enrolment as part of the initial set of evaluations. The standard of care cervical MRI is not required to be initiated prior to the start of glyburide. Glyburide will, therefore, be administered as soon as possible following enrolment irrespective of whether the MRI has been performed.

\section{Study drug description, dose and administration}

Enrolled patients receive 12 doses of glyburide starting within 8 hours of acute cervical tSCI. This injury-to-drug time window appears appropriate given that Sur1-Trpm 4 receptor expression was first noted to be increased at 6 hours following injury in a rodent tSCI model ${ }^{4}$ and will apply to patients managed non-operatively as well as to patients undergoing surgery (likely a majority of patients in the trial). The oral dosing regimen is less than that typically administered to hyperglycaemic
- $3.125 \mathrm{mg}$ : first dose of $1.25 \mathrm{mg}$ to be administered within 8 hours of $\mathrm{tSCl}$ followed by $0.625 \mathrm{mg}$ every 6 hours

- Blood Glucose (BG) to be monitored every hour

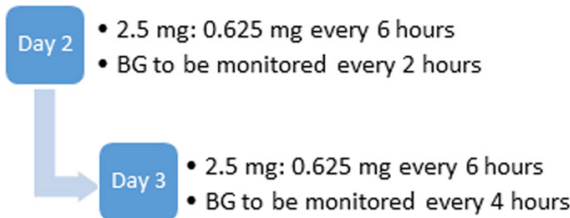

Figure 2 Flow diagram depicting glyburide dosing and BG monitoring schedule over 3 days following enrolment. $\mathrm{tSCl}$, traumatic spinal cord injury.

patients following stroke or traumatic brain injury ${ }^{31}$ and parallels the intravenous dosing employed in the stroke Glyburide Advantage in Malignant Edema and Stroke (GAMES-RP) trials. ${ }^{23} 24$ The regimen involves an initial dose of $1.25 \mathrm{mg}$, followed by 11 consecutive doses of $0.625 \mathrm{mg}$ every 6 hours. The total daily dose of glyburide on day 1,2 and 3 is $3.125,2.5$ and $2.5 \mathrm{mg}$, respectively (see figure 2).

Drug administration should not be stopped as the standard of care diagnostic studies and treatment is provided, including if the patient is transferred to the operating room for decompression and stabilisation. In the operating room or in the event of any swallowing difficulty, glyburide will be administered via a nasogastric tube. In the event a dose of glyburide is missed (beyond 2.5 hours from the expected administration time), the next dose is administered at $1.25 \mathrm{mg}$, followed by the usual $0.625 \mathrm{mg}$ dose for subsequent administrations. If the missed dose is acknowledged within 2.5 hours of the expected administration time, the dose is given and the deviation noted.

Glyburide dosing is reduced to every 8 hours if the subject is being administered D5NS or D10NS in response to a hypoglycaemic reading and (1) there is one confirmed $\mathrm{BG}<55 \mathrm{mg} / \mathrm{dL}$; or (2) there are three confirmed $\mathrm{BG}<70 \mathrm{mg} / \mathrm{dL}$ within a 12-hour period. If appropriate levels of $\mathrm{BG}$ are not reached within 8 hours of the dose reduction, the next dose of glyburide is skipped as an additional dose reduction. If (1) or (2) above occurs a second time, glyburide is discontinued. When glyburide is stopped as a result of low BG, D50W is administered by bolus in order to maintain $\mathrm{BG}>80 \mathrm{mg} / \mathrm{dL}$.

\section{Prohibited interventions and interventions related to $B G$}

Sulfonylurea agents and Insulin for 'tight' BG control (when BG less than $120 \mathrm{mg} / \mathrm{dL}$ ) are not permitted during the period of glyburide administration. Supplemental fluids are to be standard of care except that during glyburide administration, D5NS or D10NS must be used instead of NS if necessary to maintain BG above $80 \mathrm{mg}$ / $\mathrm{dL}$. Any BG of less than $70 \mathrm{mg} / \mathrm{dL}$ is promptly treated with a bolus of D50W at a volume (mL) of (100-BG in $\mathrm{mg}$ / dL) x 0.4. ${ }^{32}$ If supplemental fluids are NS, either D5NS or D10NS should be started. 


\section{Post-treatment follow-up}

Post-treatment follow-up assessments will be undertaken at day 28, 42 and 84 ( \pm 7 days) with review of unresolved AEs, record additional AEs and ISNCSCI assessments. Post-treatment follow-up assessments will also be undertaken at day 84 ( \pm 14 days), day $182( \pm 14$ days) and day 365 ( \pm 30 days) with additional assessment of SCIM version III. Patients will be contacted in advance by mail and phone to communicate and schedule clinical assessments. All data will be entered and managed using a Research Electronic Data Capture database. Electronic monitoring and source documentation verification will occur following each patient enrolment and every 6 months thereafter up to 1 year. To protect personal health information belonging to both screened and enrolled patients, all data regarding the SCING study will be stored on encrypted drives in The Ohio State University Wexner Medical Center Department of Neurological Surgery Research Department. All data will only be accessible to properly trained personnel approved by the IRB. Data collected will be deidentified or coded whenever possible. Any keys containing direct identifiers between coded data sets will be stored separately from the coded set.

\section{AEs and SAEs}

In this study, AEs will be continually monitored through day 14 or up until discharge (whichever is earlier). AEs will be further reviewed and additional AEs recorded on day 28 ( \pm 7 days). Minimum information required for each AE includes type of event, duration (start and end dates), severity (mild, moderate, severe), seriousness (AE vs SAE), causality to glyburide, action taken and outcome. The relationship of the AE to glyburide will be specified as either unrelated, possibly/probably related and definitely related. AEs will be followed for resolution through the end of the study. Beyond day 28 through the end of the study, only new SAEs will be recorded.

\section{Stopping rules: criteria for intervention discontinuation}

Glyburide is to be discontinued if the dose has already been reduced in accordance with the defined guidelines and following the reduction (1) there is one laboratory or repeat point-of-care confirmed $\mathrm{BG}<55 \mathrm{mg} / \mathrm{dL}$ or (2) there are three laboratories or repeat point-of-care confirmed $\mathrm{BG}<70 \mathrm{mg} / \mathrm{dL}$ within a 12-hour period. Glyburide is also stopped if a life-threatening cardiac-related SAE occurs and/or the subject experiences QTc of $>550 \mathrm{~ms}$ (Bazett's formula) for $15 \mathrm{~min}$, in either circumstance whether or not believed by the clinician to be related to Glyburide. Finally, glyburide is discontinued if alanine transaminase raises to greater than eightfold the upper limit of normal or if the subject develops either cholestatic jaundice or hepatitis.

\section{Data and safety monitoring board}

The data and safety monitoring plan involves the continuous evaluation of safety, data quality and data timeliness. Investigators will conduct continuous review of accrual and patient safety. The frequency and severity of AEs will be reviewed by the study investigators and compared with other sources, including published literature and scientific meetings. All AEs and SAEs will also be reviewed by the SCING data and safety monitoring board (DSMB), which consists of three independent members including a neurosurgeon, a biostatistician and a clinical trial specialist. All DSMB members reviewed the trial protocol prior to study activation in order to ensure appropriate implementation of safety procedures and endpoints. DSMB meetings are scheduled within a month of every third patient enrolled in the study. The study investigators in conjunction with the DSMB will determine whether the trial should be terminated early based on the AE reviews.

\section{Statistical considerations}

The frequency of AEs will be reported and, where possible, will be compared with those of the matched controls (along with corresponding confidence intervals). Study patients and the historical NACTN cohort data will be matched on criteria including: age, comorbidities, injury level and injury severity (AIS A, B or C). Additionally, a number of patients who were otherwise eligible for the study but did not enrol due to lack of administration of glyburide within 8 hours will be reported. Secondarily, matched tests will be used to compare observed and expected neurological outcomes as based on our recent prediction analysis. ${ }^{14}$ The statistical plan will involve cautious interpretation of descriptive and non-parametric analyses.

\section{Patient and public involvement}

There was no patient or public involvement in the design or implementation of this pilot study. Participants enrolled in the trial will be included in discussions regarding their response to the study treatment as well as their long-term neurological outcomes. In preparation for subsequent phases, patient and public involvement with be required to determine the optimal design under exception from informed consent (EFIC) guidelines. The planned prestudy community consultation and public notification will allow patients and the public a platform to ask specific questions, provide input with new ideas and potentially opt out of participation. Subsequent study designs involving a blinded randomisation component will allow patients to be unblinded once patient enrolment and follow-up is completed. This information will be shared with patients through the contact method of their preference (documented phone conversation or verified letter). Additionally, any safety information discovered during the course of this and subsequent phases will be shared with participants per Food and Drug Administration (FDA) guidelines.

\section{ETHICS AND DISSEMINATION}

Glyburide has been shown as an effective neuroprotective agent in preclinical models and in the treatment of 
ischaemic stroke with the additional risk of a hypoglycaemic response. Given the ongoing secondary injury and the traumatic hyperglycaemic stress response seen in patients with tSCI, glyburide, thus, offers an appealing neuroprotective strategy to supplement standard of care treatment.

Participants or legally authorised representatives are informed about the trial and its anticipated risks orally and in written form using an approved informed consent form prior to inclusion. The study complies with the Declaration of Helsinki and the principles of Good Clinical Practice. The findings of this study will be disseminated to participants and to academic peers through scientific conferences and peer-reviewed journal publications. All investigators that contributed to study conduction and analyses will be included as coauthors on all published materials.

\section{DISCUSSION}

\section{Clinical need for novel neuroprotective strategies following tSCI}

There are currently no proven neuroprotective strategies for the treatment of acute tSCI. While once considered as standard of care for patients with acute tSCI, it is noteworthy that more recent recommendations have re-evaluated the routine use of methylprednisolone. ${ }^{33}$ The most recent guidelines published by a panel convened by the Joint Section on Spine and Peripheral Nerves of the American Association of Neurological Surgeons and Congress of Neurological Surgeons has in fact recommended against its use following acute tSCI given its negative risk-benefit profile. ${ }^{26}$ In the words of the panel: 'Administration of methylprednisolone for the treatment of acute SCI is not recommended. Clinicians considering methylprednisolone therapy should bear in mind that the drug is not approved by the FDA for this application. There is no class I or class II medical evidence supporting the clinical benefit of methylprednisolone in the treatment of acute SCI. Scattered reports of class III evidence claim inconsistent effects likely related to random chance or selection bias. However, class I, II and III evidence exists that high-dose steroids are associated with harmful side effects, including death.' Of note, a recent phase 1/2a study evaluating the sodium-channel blocker Riluzole reported that corticosteroids were administered at the time of admission to only $39 \%$ of the patients (while $58 \%$ of historic NACTN registry patients had received this treatment). ${ }^{30}$ Given that safety data appeared favourable in this early phase study, a larger phase 2/3 study is currently underway to assess for efficacy in improving neurological outcomes (RISCIS Study, NCT01597518). Other potential therapies are either at the preclinical or early pilot stage. ${ }^{34}$

Progressive haemorrhagic necrosis (PHN) represents an increasingly well-characterised mechanism of secondary injury that negatively impacts neurological outcomes. Structural failure of the integrity of intramedullary capillaries, known as 'capillary fragmentation', is thought to underlie this acute and dynamic secondary injury process. Animal studies modelling contusional SCIs across different laboratories have revealed a 2 to 2.5 -fold increase in extravasated intramedullary blood within the first 12-24 hours following blunt impact trauma. ${ }^{435} 36$ The formation of distinct microscopic haemorrhages within the cord is in turn linked to the progressive secondary injury and neurological deterioration following SCI.

\section{Glyburide and signalling background}

Numerous pharmacokinetic studies of oral glyburide have assessed single-dose administrations in normal subjects and have demonstrated significant absorption within 1 hour, peak drug levels at about 4 hours and low but detectable levels at 24 hours. Mean serum levels of glyburide, as reflected by areas under the serum concentration-time curve, increase in proportion to corresponding increases in dose. Multiple-dose studies with oral glyburide in patients with diabetes demonstrate drug-level concentration-time curves similar to singledose studies, indicating no build-up of drug in tissue depots. The decrease of glyburide in the serum of normal healthy individuals is biphasic, the terminal half-life being about 10 hours. $^{20}$

In a phase 1 study of intravenous glyburide (Cirara), the drug was administered as a bolus dose, followed by a 3-day continuous infusion maintenance dose. Five groups of patients were dosed, totalling 26 patients on drug (four dosing groups, with doses ranging from 0.4 to $10 \mathrm{mg}$ / day) and eight on placebo. There were no SAEs observed during this study. Two subjects (one in the $6 \mathrm{mg} /$ day treatment group and one in the $10 \mathrm{mg}$ /day treatment group) were discontinued from the study due to persistent hypoglycaemia. The subject in the $6 \mathrm{mg}$ /day treatment group who was discontinued from the study also experienced transient increases in liver enzyme levels beginning 3 days after study drug discontinuation, which returned to normal by day 22. There were no other clinically significant drug-related adverse reactions. ${ }^{37}$ Based on this work, phase 2a pilot and exploratory randomised trials were undertaken (GAMES-RP) wherein a total of 93 stroke patients were dosed at $3 \mathrm{mg} /$ day for 3 days. There were no new safety concerns raised in these patients. ${ }^{23}$

\section{Rationale for glyburide following tSCI}

Emerging evidence implicates the Sur1-Trpm 4 channel as the molecular precursor of PHN. ${ }^{4-9}$ Upregulation and coassociation of the regulatory and pore forming subunits of this ion channel have been demonstrated to occur in endothelial and other cells after tSCI, as demonstrated in rodents and humans. ${ }^{41038} \mathrm{PHN}$-associated secondary microhaemorrhage formation in turn is specifically associated with channel upregulation and activation in micro vessels at 6 hours following tSCI in rodents with expanding expression noted up to 24 hours. ${ }^{4039}$ Following trauma, spinal cord oedema and microhaemorrhage formation through this mechanism is a critical secondary injury 
process that exacerbates mechanical compression and ischaemia, and contributes to neuronal/glial cell death.

Glyburide-mediated blockade of the Sur1-Trpm4 channel in rodent models of tSCI leads to minimal secondary microhaemorrhage formation and the absence of capillary fragmentation, the pathological hallmark of PHN. Lesion volumes and hindlimb locomotor functional outcome measures, measured up to 6 weeks, are significantly improved in animals treated with glyburide shortly after the time of injury. ${ }^{4-10}$ Delayed treatment at 3-4hours following tSCI in rats, a more clinically relevant time frame, also yielded significant functional benefit. ${ }^{40} 41$ While the magnitude of glyburide-mediated neurological benefit appears to correlate with the magnitude of the initial experimental injury, significant treatment effects have been consistently demonstrated for both lesion volume and functional scores. ${ }^{9} 1041$

\section{Study limitations and future prospects}

Between August 2015 and August 2016, we screened patients with acute cervical tSCI (GOSCIP study; clinical trials NCT02524379) for potential treatment with an intravenous formulation of glyburide (Cirara). Fourteen patients were screened during this period but all failed to meet the eligibility criteria. Given the ongoing enrolment difficulty with this intravenous drug within the 6-hour enrolment window, we decided to amend several elements of the trial including some of the inclusion and exclusion criteria. First, the route of administration was changed to the oral form allowing for easier and streamlined drug administration. Although no studies to date have assessed comparative drug route-based pharmacokinetics following trauma, no significant difference in total metabolite urine recovery between intravenous or oral glyburide has been noted in normal human subjects, suggesting identical bioavailability of the two formulations. ${ }^{42}$ An extension of the window of administration to 8 hours from the time of injury was also implemented. This injury-to-drug time window still appears appropriate given that Sur1-Trpm4 receptor expression is first noted to be increased at 6 hours following injury in a rodent tSCI model. ${ }^{4}$ We estimated that 5 out of 14 screened patients fell out of potential eligibility due to this constrained time window, especially considering that Cirara preparation at our principal site was noted to take in the order of 1-2 hours.

Given glyburide's well known mechanism of action, the potential for hypoglycaemia in non-diabetic patients could be viewed as a limitation of this study design. Nevertheless, neurotrauma patients, including those with tSCIs, typically have elevated BG levels over the first 3 days following their injury. For example, a large retrospective review of 528 patients with $\mathrm{tSCI}^{43}$ indicated that $53 \%$ of patients were hyperglycaemic on admission (glucose concentration $\geq 126 \mathrm{mg} / \mathrm{dL}$ ) and that hyperglycaemia was a significant risk predictor of poor functional outcome. As such, oral glyburide administration, for its potential neuroprotective effects in the tSCI population, appears unlikely to cause significant AEs related to hypoglycaemia and may even be independently beneficial given its BG lowering effects. A double-blind, multicentre, randomised trial of an intravenous form of glyburide (Cirara) in patients with large ischaemic strokes likely to develop malignant cerebral oedema (GAMES-RP study), identified the drug to be well tolerated at $3 \mathrm{mg}$ per day ${ }^{23}$ with few treatment-related hypoglycaemia SAEs (4 events in 44 patients) managed with glucose supplementation. While the oral dosing of glyburide used in this study may theoretically increase the risk of hypoglycaemic episodes as compared with continuous intravenous infusion, we have not noted any such episodes thus far in the initial enrolled patients. Careful continued BG and AE monitoring will be undertaken to assess for the relative importance of the post-tSCI stress response vs the hypoglycaemia-inducing property of glyburide.

Despite the protocol amendments, enrolment has remained modest with only two subjects at our three centres. While likely related to multiple causes, the narrow post-tSCI time window for Glyburide administration has remained a critical element that has restricted enrolment. As a result, in preparation for the next potential exploratory trial phase, we are currently evaluating the option of administering glyburide under (EFIC; 21 Code of Federal Regulations \$50.24) guidelines, ${ }^{44}$ which would need to be undertaken with patient/public consultation and in collaboration with emergency medical services. While time-consuming and expensive, the stroke research field has ultimately benefited significantly from EFIC trials. ${ }^{45-49}$

The absence of reliable tools to assess injury severity on admission and to predict the likelihood of neurological recovery has markedly complicated tSCI clinical trial design. The noted heterogeneity in outcomes has particularly confounded the evaluation of potential neuroprotective agents that depend on immediate-to-early administration. As a result, numerous multicentre trials have struggled to achieve the relatively higher required recruitment numbers, often planned over constricted timelines ${ }^{50}$ In view of the critical importance of improved patient stratification and prognostication, we previously reviewed 99 consecutive patients with acute cervical tSCI at our principal site (table 1) to comparatively assess surrogate imaging biomarkers with respect to their ability to predict neurological outcomes in the chronic recovery phase after tSCI. ${ }^{14}$ As compared with other parameters, we confirmed that the axial imaging-based admission BASIC score is the single best predictor of AIS conversion at 1 year after tSCI. As such, we anticipate that incorporation of admission imaging findings for improved tSCI prognostication will ultimately allow for a more reliable assessment of the potential efficacy of oral Glyburide, particularly in subsequent larger exploratory trial phases.

The SCING pilot phase trial represents a small and open-label study by design, therefore necessitating cautious interpretation. The clinical trial findings will be reported on completion of enrolment as a publication in 
a peer-reviewed journal irrespective of the primary and secondary outcome results. The study's findings may provide valuable data on glyburide's safety and pharmacokinetic profile as well as preliminary data on its ability to improve neurological outcomes and surrogate biomarkers following tSCI. Taken together, these data will allow for a more rational subsequent phase II trial design.

\section{Author affiliations}

${ }^{1}$ Department of Neurological Surgery, Ohio State University Wexner Medical Center, Columbus, Ohio, USA

${ }^{2}$ Department of Neurosurgery, University of Illinois at Chicago, Chicago, Illinois, USA ${ }^{3}$ Department of Neurological Surgery, Loma Linda University, Loma Linda, California, USA

${ }^{4}$ Department of Neurology, Ohio State University Wexner Medical Center, Columbus, Ohio, USA

${ }^{5}$ Department of Neurological Surgery, University of Maryland School of Medicine, Baltimore, Maryland, USA

Contributors All authors participated in critically revising and reviewing the submitted version of the manuscript. HFF and AJM drafted the article. HFF, AJM and JMS conceived and designed the study and manuscript. Acquisition of data and study supervision is performed by HFF, AJM, AM and WWB. Overall analysis and interpretation was performed by AJM, HFF and JMS.

Funding Internal funding support is provided by the Department of Neuological Surgery at The Ohio State University Wexner Medical Center.

Competing interests JMS reports personal fees from Remedy Pharmaceuticals, personal fees from Biogen, outside the submitted work. In addition, JMS has a US patent $(\# 7,872,048)$, 'Methods for treating spinal cord injury with a compound that inhibits a NC(Ca-ATP) channel' with royalties paid to University of Maryland.

Patient consent for publication Not required.

Ethics approval The SCING study protocol was approved by the Ohio State University Biomedical Institutional Review Board.

Provenance and peer review Not commissioned; externally peer reviewed.

Open access This is an open access article distributed in accordance with the Creative Commons Attribution Non Commercial (CC BY-NC 4.0) license, which permits others to distribute, remix, adapt, build upon this work non-commercially, and license their derivative works on different terms, provided the original work is properly cited, appropriate credit is given, any changes made indicated, and the use is non-commercial. See: http://creativecommons.org/licenses/by-nc/4.0/.

\section{ORCID iD}

H Francis Farhadi http://orcid.org/0000-0002-4668-8512

\section{REFERENCES}

1 Chen M, Dong Y, Simard JM. Functional coupling between sulfonylurea receptor type 1 and a nonselective cation channel in reactive astrocytes from adult rat brain. J Neurosci 2003;23:8568-77.

2 Chen M, Simard JM. Cell swelling and a nonselective cation channel regulated by internal $\mathrm{Ca}^{2+}$ and ATP in native reactive astrocytes from adult rat brain. J Neurosci 2001;21:6512-21.

3 Simard JM, Chen M, Tarasov KV, et al. Newly expressed SUR1regulated NCCa-ATP channel mediates cerebral edema after ischemic stroke. Nat Med 2006;12:433-40.

4 Simard JM, Tsymbalyuk O, Ivanov A, et al. Endothelial sulfonylurea receptor 1-regulated NCCa-ATP channels mediate progressive hemorrhagic necrosis following spinal cord injury. J. Clin. Invest. 2007;117:2105-13.

5 Simard JM, Kent TA, Chen M, et al. Brain oedema in focal ischaemia: molecular pathophysiology and theoretical implications. Lancet Neurol 2007;6:258-68.

6 Simard JM, Woo SK, Schwartzbauer GT, et al. Sulfonylurea receptor 1 in central nervous system injury: a focused review. J Cereb Blood Flow Metab 2012;32:1699-717.

7 Popovich PG, Lemeshow S, Gensel JC, et al. Independent evaluation of the effects of glibenclamide on reducing progressive hemorrhagic necrosis after cervical spinal cord injury. Exp Neurol 2012;233:615-22.
8 Simard JM, Popovich PG, Tsymbalyuk O, et al. Mri evidence that glibenclamide reduces acute lesion expansion in a rat model of spinal cord injury. Spinal Cord 2013;51:823-7.

9 Simard JM, Popovich PG, Tsymbalyuk O, et al. Spinal cord injury with unilateral versus bilateral primary hemorrhage - effects of glibenclamide. Exp Neurol 2012;233:829-35.

10 Simard JM, Woo SK, Norenberg MD, et al. Brief suppression of ABCC8 prevents autodestruction of spinal cord after trauma. Sci Transl Med 2010;2:28ra29.

11 Grossman RG, Frankowski RF, Burau KD, et al. Incidence and severity of acute complications after spinal cord injury. J Neurosurg 2012;17(Suppl 1):119-28.

12 Grossman RG, Toups EG, Frankowski RF, et al. North American clinical trials network for the treatment of spinal cord injury: goals and progress. J Neurosurg 2012;17(Suppl 1):6-10.

13 Catz A, Itzkovich M. Spinal cord independence measure: comprehensive ability rating scale for the spinal cord lesion patient. $J$ Rehabil Res Dev 2007;44:65-8.

14 Farhadi HF, Kukreja S, Minnema A, et al. Impact of admission imaging findings on neurological outcomes in acute cervical traumatic spinal cord injury. J Neurotrauma 2018;35:1398-406.

15 Talbott JF, Whetstone WD, Readdy WJ, et al. The brain and spinal injury center score: a novel, simple, and reproducible method for assessing the severity of acute cervical spinal cord injury with axial T2-weighted MRI findings. J Neurosurg 2015;23:495-504.

16 Kuhle J, Gaiottino J, Leppert D, et al. Serum neurofilament light chain is a biomarker of human spinal cord injury severity and outcome. $J$ Neurol Neurosurg Psychiatry 2015;86:273-9.

17 Kwon BK, Casha S, Hurlbert RJ, et al. Inflammatory and structural biomarkers in acute traumatic spinal cord injury. Clin Chem Lab Med 2011;49:425-33.

18 Yokobori S, Zhang Z, Moghieb A, et al. Acute diagnostic biomarkers for spinal cord injury: review of the literature and preliminary research report. World Neurosurg 2015;83:867-78.

19 Aarabi B, Simard JM, Kufera JA, et al. Intramedullary lesion expansion on magnetic resonance imaging in patients with motor complete cervical spinal cord injury. J Neurosurg 2012;17:243-50.

20 sanofi-aventis US LLC. DiaBeta package insert. Bridgewater: sanofiaventis US LLC, 2009.

21 Kopp MA, Liebscher T, Watzlawick R, et al. SCISSOR-Spinal cord injury study on small molecule-derived Rho inhibition: a clinical study protocol. BMJ Open 2016;6:e010651.

22 Lammertse D, Tuszynski MH, Steeves JD, et al. Guidelines for the conduct of clinical trials for spinal cord injury as developed by the ICCP panel: clinical trial design. Spinal Cord 2007;45:232-42.

23 Sheth KN, Elm JJ, Molyneaux BJ, et al. Safety and efficacy of intravenous glyburide on brain swelling after large hemispheric infarction (GAMES-RP): a randomised, double-blind, placebocontrolled phase 2 trial. Lancet Neurol 2016;15:1160-9.

24 Sheth KN, Kimberly WT, Elm JJ, et al. Pilot study of intravenous glyburide in patients with a large ischemic stroke. Stroke 2014;45:281-3.

25 Wilson JR, Vaccaro A, Harrop JS, et al. The impact of facet dislocation on clinical outcomes after cervical spinal cord injury: results of a multicenter North American prospective cohort study. Spine 2013;38:97-103.

26 Walters BC, Hadley MN, Hurlbert RJ, et al. Guidelines for the management of acute cervical spine and spinal cord injuries. Neurosurgery 2013;60(Suppl 1):82-91.

27 Fehlings MG, Vaccaro A, Wilson JR, et al. Early versus delayed decompression for traumatic cervical spinal cord injury: results of the Surgical Timing in Acute Spinal Cord Injury Study (STASCIS). PLoS One 2012;7:e32037.

28 Galer BS, Jensen MP. Development and preliminary validation of a pain measure specific to neuropathic pain: the neuropathic pain scale. Neurology 1997;48:332-8.

29 Widerström-Noga E, Biering-Sørensen F, Bryce TN, et al. The International spinal cord injury pain extended data set (version 1.0). Spinal Cord 2016;54:1036-46.

30 Grossman RG, Fehlings MG, Frankowski RF, et al. A prospective, multicenter, phase I matched-comparison group trial of safety, pharmacokinetics, and preliminary efficacy of riluzole in patients with traumatic spinal cord injury. J Neurotrauma 2014;31:239-55.

31 Pallan TV, Ahmed I. Glyburide in treating malignant cerebral edema. blocking Sulfonyl Urea One (SUR1) receptors. J Vasc Interv Neurol 2014;7:23-5.

32 Juneja R, Roudebush CP, Nasraway SA, et al. Computerized intensive insulin dosing can mitigate hypoglycemia and achieve tight glycemic control when glucose measurement is performed frequently and on time. Crit Care 2009;13. 
33 Evaniew N, Noonan VK, Fallah N, et al. Methylprednisolone for the treatment of patients with acute spinal cord injuries: a propensity score-matched cohort study from a Canadian multi-center spinal cord injury registry. J Neurotrauma 2015;32:1674-83.

34 Ahuja CS, Fehlings M. Concise review: bridging the gap: novel neuroregenerative and neuroprotective strategies in spinal cord injury. Stem Cells Transl Med 2016;5:914-24.

35 Balentine JD. Pathology of experimental spinal cord trauma. I. The necrotic lesion as a function of vascular injury. Lab Invest 1978;39:236-53.

36 Bilgen M, Abbe R, Narayana PA. Dynamic contrast-enhanced MRI of experimental spinal cord injury: in vivo serial studies. Magn. Reson. Med. 2001;45:614-22.

37 Simard JM, Sheth KN, Kimberly WT, et al. Glibenclamide in cerebral ischemia and stroke. Neurocrit Care 2014;20:319-33.

38 Woo SK, Kwon MS, Ivanov A, et al. The sulfonylurea receptor 1 (Sur1)-transient receptor potential melastatin 4 (Trpm4) channel. J Biol Chem 2013;288:3655-67.

39 Gerzanich V, Woo SK, Vennekens R, et al. De novo expression of TRPM4 initiates secondary hemorrhage in spinal cord injury. Nat Med 2009;15:185-91.

40 Hosier H, Peterson D, Tsymbalyuk O, et al. A direct comparison of three clinically relevant treatments in a rat model of cervical spinal cord injury. J Neurotrauma 2015;32:1633-44.

41 Simard JM, Tsymbalyuk O, Keledjian K, et al. Comparative effects of glibenclamide and riluzole in a rat model of severe cervical spinal cord injury. Exp Neurol 2012;233:566-74.
42 Rydberg T, Jönsson A, Melander A. Comparison of the kinetics of glyburide and its active metabolites in humans. J Clin Pharm Ther 1995;20:283-95.

43 Kobayakawa K, Kumamaru $\mathrm{H}$, Saiwai $\mathrm{H}$, et al. Acute hyperglycemia impairs functional improvement after spinal cord injury in mice and humans. Sci Transl Med 2014;6:256ra137.

44 Research USDoHaHSCfDEa. Guidance for institutional review boards, clinical Investigators, and sponsors exception from informed consent requirements for emergency research, 2013.

45 Bateman BT, Meyers PM, Schumacher HC, et al. Conducting stroke research with an exception from the requirement for informed consent. Stroke 2003;34:1317-23.

46 Kleindorfer D, Lindsell CJ, Alwell K, et al. Ischemic Stroke Survivors' Opinion Regarding Research Utilizing Exception from Informed Consent. Cerebrovasc Dis 2011;32:321-6.

47 Ripley E, Ramsey C, Prorock-Ernest A, et al. Ems providers and exception from informed consent research: benefits, ethics, and community consultation. Prehosp Emerg Care 2012;16:425-33.

48 Rose DZ, Kasner SE. Informed consent: the rate-limiting step in acute stroke trials. Front Neurol 2011;2:65

49 Silbergleit R, Biros MH, Harney D, et al. Implementation of the exception from informed consent regulations in a large multicenter emergency clinical trials network: the RAMPART experience. Acad Emerg Med 2012;19:448-54.

50 Blight AR, Hsieh J, Curt A, et al. The challenge of recruitment for neurotherapeutic clinical trials in spinal cord injury. Spinal Cord 2019;57:348-59. 